\title{
Sausalito: An Application Servers for RESTful Services in the Cloud
}

\author{
Matthias Brantner \\ $28 \mathrm{msec} \mathrm{GmbH}$ \\ Zurich, Switzerland \\ matthias.brantner@28msec.com
}

This talk argues that Web Server, Application Server, and Database System should be bundled into a single system for development and deployment of Web-based applications in the cloud. Furthermore, this talk argues that the whole system should serve REST services and should behave like a REST service itself. The design and implementation of Sausalito is presented which is a combined Web, Application, and Database server that operates on top of Amazon's cloud offerings. Furthermore, a demo of several example applications is given that show the advantages of the approach taken by Sausalito (see http://sausalito.28msec.com/). 